demonstrated the last in a great number of cases; and some. times they form a "visible white deposit", such as Dr. Bird described. It is a pity that the name "organic globule" has been used at all, for certainly several widely different substances answer to the characters given to it. The so-called "large organic globules", or " exudation-corpuscles", have been shown to consist of an aggregation of fat-globules, such as is found in cases of fatty degeneration of the kidney. I have therefore thought it better, in order to avoid confusion, not to employ the term in these lectures. (See Lecture $\mathrm{Ix}$; also The Microscope in Medicine, 2nd ed., p. 326.)

If the practitioner should meet with objects whose nature he cannot ascertain, he should at once make careful drawings, and take notes of the case in which they occurred. The importance of being familiar with the appearances of all the extraneous substances likely to be met with has been already referred to.

[To be continued.]

\section{olinital apecture \\ on}

\section{BLOWS ON THE EYE-BALL, ATTENDED WITH ; RUPTURE OR LACERATION ; AND DISTURBANCE OF THE CONTAINED PARTS.}

By HAYNES WALTON, Esq., Surgeon to St. Mary's, and to the Central London Ophthalmic Hospitals.

Gentienen,-Blows on the globe of the eye may cause rup. ture of its coats, or the separation, or dislocation, of some of its internal parts. Bursting is no uncommon occurrence, and when the sclerotica suffers, the superior, or the internal por. tion, between the cornea and a line concentric to the attach. ment of one of the recti muscles generally gives way. I have seen but one exception, when the rupture was on the outer side. It has been suggested that the sclerotica tears at a point nearly opposite to that which is struck, the blows which reach the eye-ball being, for the most part, on its lower, or its outer side; the upper edge of the orbit and the nose protecting it in these positions. The vitreous humour may escape in greater or lesser quantity, and accompanying this may be prolapse of the retina and the choroid. With so much damage the organ is very often destroyed. In a few cases I have seen profuse hæmorrhage.

Loosening of the lens, or more commonly displacement of it, is another effect of blows on the eye and on the head. Dislocation forwards is that most usual; and it may be either alone or in the capsule. If it be thrown against the back of the iris, and does not become opaque, the nature of the accident might be temporarily overlooked. The pupil may be occupied by the lens, but rarely for any length of time, since it either falls against the iris, or, what is more likely, into the anterior chamber. l'ain is the ustal concomitant of any form of this accident. It may exist with little or no inflammatory action. Sometimes it is of an intensely neuralgic character, portending destruction of vision, not only in the eye, but about the orbit and head. Acute inflammation mostly supervenes, and the organ is destroyed. I have, in a few cases, seen low inflammatory action, with little or no pain, equally fatal. In all cases the dislocated bod 5 must be extracted, if possible; but if this be impracticable, it should be reclined.

But the leus may lie in the anterior chamber without any untoward symptoms; and, most assuredly, if no irritation were set up, I should leave it alone and trust to absorption. Even with slight local disturbance, in a patient under forty years of age, I should not interfere. Above forty, when the lens fibres increase in density, and absorption of them cannot quickly occur, I should make the earliest symptoms of irritation the signal for me to act. A dislocated lens and capsule should be removed from the chamber at once, that is as soon as we have assurance that the capsule is present, for months and years may pass away before ahsorption occurs, and even were it to be effected before the eye has been destroyed, the capsule might prove a source of much irritation, and, perhaps, become the seat of calcareous deposit. Mr. Laurence mentions the particulars of a patient who occasionally visited the Jondon Ophthalmic
Infirmary, with the lens surrounded by its capsule in the anterior chamber, where it had been for twenty-eight years; the general state of the eye is not given.

But the capsule may not get opaque; and how is its presence to be detected? I answer this by an extract from the review of my work on the eye, in the British and Foreign MedicoChirurgical Review, by Dr. MacKenzie. He says "weeks, months, and, I suspect, years, may pass before any decided opacity shows itself in the lens or capsule, provided the latter enclosing the former has been separated from its connections, without any aperture by which the entrance of the aqueous humour is permitted. In such a case, the lens shows itself in the anterior chamber like a drop of water, of different specific gravity from the rest of the aqueous humour, and its margin seems surrounded by a narrow gilt ring of a splendid yellow colour! In this state he had extracted the lens and capsule before they exhibited the least opacity, although they had been dislocated for weeks. This is a point that $I$ have fully considered in the forthcoming edition of $\mathrm{my}$ book, now in the press.

I may mention incidentally the particulars of a case of spontaneous dislocation of the lens and its capsule into the anterior chamber, in which the eye was lost by the irritation produced. An overgrown girl of thirteen was brought to me about three years ago, with staphyloma completely around and close to the cornea, enlargement and semi-opacity of the cornea, much pain, extinction of vision, and general redness of the eye-ball. While endeavouring to ascertain the cause of all this, an opaque capsule enclosing the lens passed forward into the anterior chamber. I now learnt that for some months this opaque body had been observed to pass from one chamber of the eye to the other. It was at once apparent that all the morbid action was produced by the dislocated part. As the other eye was suffering from supposed sympathetic implication, it was examined carefully,'and its lens and capsule, quite opaque, were found to be reclining backwards into the vitreous humour, but yet retained a slight attachment to the lower portion of the suspensory ligament. The necessary operation on the right eye, that of "extraction," was followed by a cessation of all irritation. The parents of the girl would not allow the other eye to be touched.

Mr. Henry Howard relates, in one of the periodicals, an instance of spontaneous dislocation of lens into the anterior chamber of the eye, being productive of cerebral derangement. A female, aged 30 , suffered from pain and inflammation in her left eye for nearly seven months, during the latter three of which headaches, attending with vomiting, were so severe as to deprive her of her senses. Mr. Howard found the whole eyeball inflamed, and the lens, with a small quantity of $1 \mathrm{ymph}$, lying in the anterior chamber. Extraction was performed; from that time the vomiting ceased, and the head symptoms gradually subsided, and were quite lost after forty-eight hours. The lens must have been contained in its capsule. I am inclined to think that it escaped from its position so enclosed, and not, as Mr. Howard thinks, without it, in consequence of destruction of the capsule by inflammation. It is likely enough that there was softening and giving way of the suspensory ligament.

Many instances of spontaneous dislocation, some in young people, and which are, of course, always associated with morbid changes in the eye, are recorded by the late Mr. Dalrymple and others.

Sometimes there is a rent in the sclerotica, while the conjunctiva remains intact, or but slightly torn; the crrstalline lens is forced out of the eye, and rests between these in close proximity to the cornea, constituting what is called " external dislocation of the lens." With this the iris is more or less paralysed or torn, and a portion, I believe, always carried out with the lens. Much blood is effused within the eyeball, yet vision may be retained.

The removal of the lens is the proper course. The simple process of raising the conjunctiva, snipping it, and removing the body is easily effected. It has been suggested not to interfere till a fortnight after the accident, that an opportunity may be afforded for the healing of the sclerotica; and that a simple rupture thereby may not be converted into a compound one, and the chances of recovery perhaps materially diminished. Certainly this would not be advisable if the pressure of the lens was productive of pain or irritation. In two cases I tried the method, but I conferred no benefit on my patients. In one, a young adult female, at the end of a fortnight the lens had become decomposed, and now was revealed the cause of the undue irritation, which before was unaccountable. In the other 
there was continual pain. Henceforth, I shall do that which appears to me more consonant with sound surgery, viz., to get rid of the extraneous body as soon as it is practicable; in fact, to appiy here the rule that is absolute, regarding the removal of extraneous matters from other parts of the body.

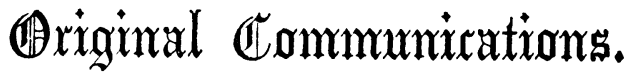

\section{TEN YEARS OF OPERATIVE SURGERY IN THE PROVINCES.}

By Augustin Prichard, Esq., Surgeon, Clifton, Bristol.

\section{IV.-Operations on the Genito.Urinary Apparatus. [Continued from page 974.]}

Stone Cases. This last sub-division of the operations in the genito-urinary system includes lithotomy and lithotrity, and I shall describe them in the following order, viz.: cases of the lateral operation, of the median section (Allarton's), litho. trity, and two cases in the female subject.

CASE CXirix. A. C., aged 11, had been troubled with symptoms of stone all his life, and occasionally had passed small bits of gravel. His symptoms were severe when I saw hin, and he had considerable hæmaturia. I performed the lateral operation in the usual way, having given him a dose of castor oil in the previous day, and removed an oxalate of lime calculus weighing a hundred grains. The operation was com. pleted in a minute and a half. He went on without any bad symptoms of importance, the wound was entirely healed in a month, and he was discharged cured.

CASE CI. C. P., aged 8, was suffering severely from the symptoms of stone when I saw him. I performed the lateral operation speedily and easily, and removed a lithic acid stone weighing seven drachms. The water flowed through the wound until the fifth day, when it began to come by the urethra. $\mathrm{He}$ was cured in little more than a fortnight.

CASE CII. E. H., also aged 8 , was under my care at the same time as the last patient, and I operated upon him on the same day, and removed a very small stone. During the whole of the next day the water flowed through the urethra, but afterwards through the wound. He went out well in about six weeks. The stone was oxalate of lime.

In the first of these two last cases, the stone was very large for so young a subject; and in the second, it was very small; but both recovered perfectly well, and in about the same time.

CASE CLII. B. G., aged 7 , with very severe symptoms of stone. In this operation I entered the groove in the staff at the first cut, and the second penetrated the bladder in the usual lateral method. I removed a large soft phosphatic calculus weighing six drachms. The stone crumbled under the forceps, and I therefore washed out the blood afterwards, and, as there was tolerably free hæmorrhage, I introduced a portion of a gum elastic catheter, and plugged the wound around it with sponge. This stopped the bleeding at once. He went out cured in about four weeks.

The catheter surrounded by the sponge was of very great use in this case, for it stopped the flow of blood, and enabled him to keep the bed dry, by letting the end of the catbeter rest in a dish.

$\mathrm{C}_{\mathrm{ASE}}$ CIII. E. H., aged 34 , a ricketty and strumous little barber, had suffered more or less from stone symptoms all his life, and for five years before I saw him they had been very severe. 'The stone was very easily discovered by the sound. I tried to crush it, but could only touch it with the points of the blades of the lithotrite, and, therefore, was obliged to perform lithotomy.

I used a large straight staff, and cut down at once upon the groove, but when I made the next cut, which should have entered the bladder, I found that it did not pass freely; in fact, the staff had slipped out of the bladder. 'This was fortunately remedied at once by passing a curved staff instead of the other, and it entered readily, and I completed the incision of the prostate. The finger having been introduced into his bladder, the stone did not fall down as usual upon it, although it was, in sounding, always felt near the neck of the bladder, and always in the same spot.

There was extreme difficulty in laying hold of it, and it could only be done by getting an assistant to press very forcibly with the hands in the hypogastric region, and when I had hold of $i$ with the forceps I could neither rotate it nor move it. I therefore broke it with the strong forceps with a screw handle, and extracted it piecemeal. It was evidently adherent at the anterior part. He lost a good deal of blood. The stone was phosphate of lime and magnesia and ammonia, with a nucleus of oxalate of lime, and an external layer of phosphate of ammonia and magnesia, that is, a mulberry calculus inside, then fusible calculus, and then externally triple phosphate, and it weighed twenty drachms and a half. He was nearly an hour under chloroform on the table. As he recovered, which he did without any very troublesome symptoms that could be referred to the operation, he suffered from constipation for sixteen days, and this yielded to an injection of ox.gall, after nearly everything else failed. No water passed through the urethra for thirty. eight days after the operation. He went out in about seven weeks, and lived well and comfortably for some time. Nearly five years have elapsed since the operation, but I heard lately that my patient has recently died.

This case was one of adhesion of a large stone to the anterior surface of the bladder, and presented very great difficulties. The great length of time which elapsed before the water came through the urethra was due to the injury inflicted in the parts by the passage of so many instruments, and the extraction of so large a stone in such rough fragments.

CASE CLIV. J. F., aged 5 years, had very severe symptoms of stone for a few weeks before his admission, having suffered altogether for eight months. He had a very narrow perineum, even for so young a child. I performed the lateral operation, and cut into his bladder withont difficulty, and in a short time extracted a lithic acid calculus weighing a hundred grains. There was less bleeding than usual. He was restless the next night, and no water passed from the wound ; and therefore, the next day, I oiled my finger and passed it through the wound, giving an escape to some urine and some small coagula. His respiration was hurried. I treated him with a little stimulus, and some opium in small doses, but he grew worse, and died on the sixth day after the operation. His most prominent symptom was cough and difficulty of breathing.

Post MORTEM Exanivation. The bladder was much thickened, looking like a small uterus; its mucous membrane was ulcerated and red in patches. There was effusion of urine between the bladder and the abdominal parietes, and into the cellular tissue of the pelvis, with a formation of unhealthy pus. There was also acute pleuro-pneumonia of both lungs; the lower lobe of the right lung sunk in water, as did separate portions of the left lung.

The cause of death here was undoubtedly an extravasation of urine into the cellular tissue of the pelvis. 'The section through the prostate did not appear to the finger to be as ex. tensive as it was in some of my former cases, particularly the first (Case cxuIX), where the patient's recovery was uninter rupted. I lost, therefore, in my cases of lateral lithotomy, one patient out of six.

Median Lithotomy. CASE CLv. W. H., aged 11 years, was admitted under my care with symptoms of stone, which had existed for nine years. I performed the median section with the fore finger of the left hand in the rectum supporting the staff, and using a sharp pointed knife, the edge directed upwards. I punctured the membranous portion of the urethra and incised it. The finger followed a probe readily through the prostate, and two stones were easily extracted. They weighed about 165 grains, and were composed of lithic acid and lithate of ammonia. The next day the boy had regained the power of holding his water, and some came through the urethra, and he went on perfectly well in every respect until the seventh day, when he had a rigor, and on the next day following an eruption like the nettle rash came out. He became rapidly worse, and died, with symptoms of pyæmia, on the eleventh day after the operation.

Post Mortem Examination. The body had begun to decompose, although the examination took place twelve hours after his death. 'The bladder was thickened, but not unhealthy. The wound in the urethra was a neat clean cut, about threequarters of an inch in length, extending as far as the prostate, but no further, and all the parts connected with the operation seemed in a satisfactory state.

That the boy died of pyæmia there was the following evidence: pus (in small quantity) in the anterior mediastinum; purulent lymph over the pleura of the right side; round deposits of thick pus in and upon both lungs; reddish pus in the left ankle joint, and between the os calcis and astragalus; a 